\title{
EVALUATION OF NEAR-PATIENT B-TYPE NATRIURETIC PEPTIDE ASSAY IN PREDICTING PATENT DUCTUS ARTERIOSUS CLOSURE IN PRETERM INFANTS ( $\leq 27+6$ WEEKS) WITH LOW DOSE INDOMETACIN PROPHYLAXIS REGIME
}

\author{
K. Jeevananthan, K. Babla, J. Paul, R. Fields, B. Jani \\ Oliver Fisher Neonatal Unit, Medway Maritime Hospital NHS Trust, Gillingham, UK
}

Background and aims: Prophylactic indometacin has been used for the prevention and closure of patent ductus arteriosis (PDA). The clinical assessment of PDA can be unreliable with echocardiography providing a more objective method of assessment, however this requires skilled personnel. Serum levels of B-type Natriuretic peptide (BNP) shows good correlation with PDA size in infants studied who have not received prophylactic indomethacin.

Our aim was to evaluate bed-side measurement of whole-blood BNP with regards to prophylactic use of 6 doses of indometacin $(0.1 \mathrm{mg} / \mathrm{kg} /$ dose/day), comparing results with colour-flow echo-cardiography findings, determining if the measurements could reliably inform the duration of the indometacin prophylaxis and clinical management.

Methods: 39 eligible babies of $\leq 27+6$ gestation born in our level 3 NICU in June 2006-February 2008 had near-patient whole-blood BNP assay and colour-flow Doppler echocardiography carried out on Days 4 and 7 of life by a single operator. This was categorised with echocardiography into open (haemo-dynamically significant), closing (non-haemo-dynamically significant) and closed (no luminal flow). The attending clinicians were blinded to the results of BNP and echocardiography.

Results:

\begin{tabular}{|c|c|c|c|c|c|c|c|c|}
\hline & $\begin{array}{c}\text { Day 4: } \\
\text { Open v } \\
\text { Closed }\end{array}$ & $\begin{array}{c}\text { Day 4: } \\
\text { Open v } \\
\text { Closing }\end{array}$ & $\begin{array}{c}\text { Day 4: } \\
\text { Open }+ \\
\text { Closing v } \\
\text { Closed }\end{array}$ & $\begin{array}{c}\text { Day 4: } \\
\text { Open v } \\
\text { Closing }+ \\
\text { Closed }\end{array}$ & $\begin{array}{c}\text { Day 7: } \\
\text { Open v } \\
\text { Closed }\end{array}$ & $\begin{array}{c}\text { Day 7: } \\
\text { Open v } \\
\text { Closing }\end{array}$ & $\begin{array}{c}\text { Day 7: } \\
\text { Open + } \\
\text { Closing v } \\
\text { Closed }\end{array}$ & $\begin{array}{c}\text { Day 7: } \\
\text { Open v } \\
\text { Closing + } \\
\text { Closed }\end{array}$ \\
\hline $\begin{array}{c}\text { Area } \\
\text { Under } \\
\text { Curve (p } \\
\text { value) }\end{array}$ & $\begin{array}{c}1.000(\mathrm{p}= \\
0)\end{array}$ & $\begin{array}{c}0.961 \\
(\mathrm{p}<0.0001)\end{array}$ & $\begin{array}{c}0.766(\mathrm{p}= \\
0.0009)\end{array}$ & $\begin{array}{c}0.972 \\
(\mathrm{p}<0.0001)\end{array}$ & $\begin{array}{c}1.000 \\
(\mathrm{p}=0)\end{array}$ & $\begin{array}{c}1.000 \\
(\mathrm{p}=0)\end{array}$ & $\begin{array}{c}0.823 \\
(\mathrm{p}<0.0001)\end{array}$ & $\begin{array}{l}1.000 \\
(\mathrm{p}=0)\end{array}$ \\
\hline $\begin{array}{c}\text { Criterion } \\
\text { Cut off } \\
\text { (pg/ml) }\end{array}$ & $>93.2$ & $>141$ & $>42.4$ & $>141$ & $>165$ & $>216$ & $>44.5$ & $>216$ \\
\hline $\begin{array}{c}\text { Sensitivity } \\
\text { (\%)/ }\end{array}$ & $100.00 /$ & $100.00 /$ & $80.65 /$ & $100.00 /$ & $100.00 /$ & $100.00 /$ & $78.26 /$ & $100.00 /$ \\
\hline $\begin{array}{c}\text { Specificity } \\
\text { (\%) }\end{array}$ & 100.00 & 94.74 & 75.00 & 96.30 & 100.00 & 100.00 & 87.50 & 100.00 \\
\hline
\end{tabular}

[Table 1: Day 4 and Day 7 PDA BNP Data Results]

Conclusions: BNP is a useful marker of ductal patency, correlating well with colour-flow echocardiography, and could be used as a valid diagnostic tool to inform the length of prophylactic indometacin treatment and further clinical management. 